\title{
Berichtigungen zu Band 94
}

p. 4, Zeile $17 / 18$ von unten: Herr Ministerialdirigent a. D. Dr. LuDwIG SCHUSTER war nicht im (Preußischen) Ministerium für Landwirtschaft, Domänen und Forsten tätig, sondern im Reichsministerium für Ernährung und Landwirtschaft.

p. 46, Zeile 16 von unten: statt "mit $14 \mathrm{~F}^{*}$ lies "mit $19 \mathrm{~F}^{*}$.

p. 53, Zeile 7 von unten: statt "daß $14 \mathrm{~F}^{*}$ Iies ${ }_{\text {}} \mathrm{daB} 19 \mathrm{~F}^{\prime}$.

p. 58, Zeile 19 von oben: statt "S.56, Tab. 3" lies "S.55, Tab. 3 .

p. 73, Zeile 1 von unten: Nicht nur $24 \mathrm{M}_{\text {t }}$ sondern auch andere in den Kurven erscheinende Männchen haben das Kontrollgebiet zeitweilig verlassen.

p. 86, Zeile 4 von oben: statt $q$ lies $\delta$.

p. 136, Zeile 14 von oben: statt "Polygynie" lies "Promiskuität". Zeile 20 von oben: siatt "polygam" lies "polygyn".

Zeile 25 von oben: statt "Polygynie" lies "Promiskuität".

p. 142, Zeile 16 von unten: statt "Polygamie" lies "Promiskuität".

p. 163. Die Unterschrift zu Abb. 1 ist folgendermaßen zu ergänzen: "Aktionsdiagramm des Paares 95 vor (3.7.52) und $\mathrm{n}$ ach (5.7.52) dem Versuch. Die schmalen Vertikalbalken bedeuten Ablösung der Gatten. Nähere Erläuterungen im Text p.162". Die zweitletzte Uhrzeit beim $O$ (14.45) muß heißen 14.55.

p. 174, Zeile 1 von oben: statt "bei einem nahe zusammen brütenden Paare" lies "bei zwei nahe zusammen brütenden Paaren".

p. 175, Zeile 14 von unten: statt "Brechungsmethoden" lies "Berechnungsmethoden". 